\title{
The number counts, luminosity functions, and evolution of microwave-selected (WMAP) blazars and radio galaxies ${ }^{\star}$
}

\author{
P. Giommi ${ }^{1,2}$, S. Colafrancesco ${ }^{1,2,3}$, P. Padovani ${ }^{4}$, D. Gasparrini ${ }^{1, \star \star, 5}$, E. Cavazzuti ${ }^{1,2}$, and S. Cutini ${ }^{1, \star \star, 5}$ \\ 1 ASI Science Data Center, ASDC c/o ESRIN, via G. Galilei, 00044 Frascati, Italy \\ e-mail: cola@mporzio.astro.it \\ 2 Agenzia Spaziale Italiana, Unità Osservazione dell’Universo, viale Liegi 26, 00198 Roma, Italy \\ on leave from: INAF - Osservatorio Astronomico di Roma, via Frascati 33, 00040 Monteporzio, Italy \\ ${ }^{4}$ European Organisation for Astronomical Research in the Southern Hemisphere (ESO), Karl-Schwarzschild-Str. 2, \\ 85748 Garching bei München, Germany \\ 5 Department of Physics, University of Perugia, via A. Pascoli, 06123 Perugia, Italy
}

Received 23 October 2007 / Accepted 28 July 2009

\section{ABSTRACT}

\begin{abstract}
We present an extensive search to identify the counterparts of all the microwave foreground sources listed in the WMAP 3-year catalogue using literature and archival data. Our work has led to the identification of 309 WMAP sources, 98\% of which are blazars, radio quasars, or radio galaxies. Only 7 WMAP detections were identified with other types of cosmic sources ( 3 starburst galaxies and 4 planetary/LBN nebulae). At present, 15 objects $(<5 \%)$ still remain without identification because of the unavailability of optical spectroscopic data or a clear radio counterpart. Our results allow us to define a flux-limited sample of 203 high Galactic latitude microwave sources $\left(f_{41 \mathrm{GHz}} \geq 1 \mathrm{Jy},\left|b_{\mathrm{II}}\right|>15^{\circ}\right)$ that is virtually completely identified (99\%). The microwave band is ideally suited to blazar statistical studies since this part of the electromagnetic spectrum is least affected by the superposition of spectral components of different origins, and therefore by selection effects. Using this data-set, we derived number counts, luminosity functions, and cosmological evolution of blazars and radio galaxies at microwave frequencies. Our results are in good agreement with those found at radio $(\mathrm{cm})$ frequencies. The $5 \mathrm{GHz}$ bivariate blazar luminosity functions are similar to those derived from the DXRBS survey, which shows that this sample is representative of the blazar population at $41 \mathrm{GHz}$. Microwave selected broad-lined quasars are about six times more abundant than BL Lacs, a ratio that is similar to, or larger than, that seen at radio and gamma-ray frequencies, once spectral selection effects are taken into account. This strongly suggests that the mechanism responsible for the generation of gammarays is, to first order, the same in all blazar types, leaving little room for models (such as external Compton radiation) that predict very different gamma-ray emission in broad-lined and lineless blazars. Our results confirm, and strengthen on a more solid statistical base, that blazars and radio galaxies are the largest contaminants of CMB anisotropy maps. We predict that these sources are also bright gamma-ray sources, most of which will be detected by the AGILE and GLAST satellites.
\end{abstract}

Key words. galaxies: active - BL Lacertae objects: general - submillimeter - radio continuum: galaxies - surveys

\section{Introduction}

Blazars are the rarest and most peculiar type of active galactic nuclei (AGN). Almost 3000 of these objects have been reported in the literature (see e.g., Massaro et al. 2009, for an updated catalogue) but this number should increase significantly in the near future as new data of previously almost unexplored energy windows (e.g., microwave, hard X-ray, gamma-ray, TeV) become available from deep astronomical observations.

Blazar observational properties typically include irregular, sometimes significant, rapid variability, apparent super-luminal motion, flat radio spectrum and large and variable polarization at radio and, especially, optical frequencies. Because of their special observational properties, blazars are assumed to be sources emitting a continuum of electromagnetic radiation from a relativistic jet that is viewed closely along the line of sight thus causing strong relativistic amplification (e.g., Blandford \& Rees 1978; Urry \& Padovani 1995).

* Table 3 is only available in electronic form at the CDS via anonymous ftp to cdsarc.u-strasbg.fr (130.79.128.5) or via http://cdsweb.u-strasbg.fr/cgi-bin/qcat?]/A+A/508/107

$\star \star$ INAF personnell resident at ASDC under ASI contract I/024/05/1
Blazars are a small fraction of all extragalactic sources but, unlike most other objects, they are strong emitters across the entire electromagnetic spectrum. In the optical and soft X-ray bands - where the radiation that we observe is mostly due to thermal emission that originated in stars and galaxies or from the accretion process onto the central engine of AGN - blazars are a tiny minority, but in other parts of the electromagnetic spectrum, where thermal emission becomes unimportant, they are often the dominant population in the extragalactic sky. Following the technological evolution and the availability of astronomical resources, most blazars have so far been discovered as counterparts of flat spectrum radio emitters or as X-ray sources. Fluxlimited samples have been compiled from several surveys in both radio and X-ray bands (e.g., Padovani et al. 2007, and references therein). Both spectral regions can, however, be affected by different selection effects: contamination by extended radio emission from the radio-lobes at $\mathrm{cm}$ wavelengths and contamination by radiation produced in the accretion process in soft X-rays, among other effects. This can be particularly important for medium to low luminosity flat spectrum radio quasars (FSRQs), where the thermal power emitted from accretion processes and non-thermal emission processes may be of comparable 
importance (e.g., Landt et al. 2008). The level of both thermal and non-thermal components can also be comparable for bright objects such as, e.g., 3C 273 (see Grandi \& Palumbo 2004).

The spectral energy distribution (SED) of blazars includes a synchrotron low-frequency component that peaks (in a $\log (v f(v))-\log (v)$ representation) between the far infrared and the X-ray band, followed by an Inverse Compton high-frequency component that has its maximum in the hard X-ray band or at higher energies (see, e.g., Giommi et al. 2007b), depending on the location of the synchrotron peak, and extends into the $\gamma$-ray or even the TeV band.

The microwave region of the electromagnetic spectrum is now available to allow for systematic studies of blazars over large cosmological volumes. This frequency band is particularly suited to the selection of blazars since at these frequencies, the contamination from radio extended components with steep spectra is no longer present and emission from the accretion process is negligible.

In this paper, we present the first flux-limited sample of microwave-selected blazars extracted from the catalogue of bright, foreground sources detected by the WMAP satellite (Bennett et al. 2003; Hinshaw et al. 2007). Using this statistically complete sample, we derive the number counts, the luminosity functions, and the cosmological evolution of both blazars and radio galaxies in the microwave band, and we compare these properties with those observed at $\mathrm{cm}$ frequencies.

Throughout this paper, source spectra are written as $S_{v} \propto v^{-\alpha}$, where $\alpha$ is the spectral index. We use a flat, vacuum-dominated CDM cosmological model with $H_{0}=$ $70 \mathrm{~km} \mathrm{~s}^{-1} \mathrm{Mpc}^{-1}, \Omega_{\mathrm{M}}=0.3$, and $\Omega_{\Lambda}=0.7$ (Spergel et al. 2006). To compare some of our results with those of previous works, we also adopt a value $H_{0}=50 \mathrm{~km} \mathrm{~s}^{-1} \mathrm{Mpc}^{-1}$ and an empty universe cosmology with $\Omega_{\mathrm{M}}=0$, and $\Omega_{\Lambda}=0$, as specifically declared in the text.

\section{The sample}

The catalogue of WMAP bright foreground sources that we consider is based on the WMAP 3-year data (Hinshaw et al. 2007) and includes 324 sources detected by WMAP in at least one channel after three years of operation. Because of the higher sensitivity of the 3-year maps, this catalogue represents a significant improvement to that based on the first year data (Bennett et al. 2003), which included 208 sources.

For the purpose of this paper, we define our flux-limited sample using source fluxes as observed in the WMAP $41 \mathrm{GHz}$ channel as a compromise between sensitivity and completeness, and the need to use the highest frequency band when defining a complete sample suitable for statistical purposes.

To define this a complete sample, we first discuss the effect of source confusion in estimating of the $41 \mathrm{GHz}$ flux of the objects that are likely to be associated with the WMAP foreground sources. We then provide a classification scheme for each object associated with the WMAP source, and finally derive the statistical properties of our complete sample.

\subsection{Source confusion}

The WMAP experiment has a limited angular resolution, which ranges between $\sim 0.93 \mathrm{deg}$ to $0.23 \mathrm{deg}(F W H M)$ for the frequency bands $23 \mathrm{GHz}$ to $94 \mathrm{GHz}^{1}$. In particular, the $41 \mathrm{GHz}$ WMAP channel, to which we refer in our study, has an angular

1 http://map.gsfc.nasa.gov/m_mm/ob_techres.html resolution of $31.8 \operatorname{arcmin}(F W H M)$. Although this limited resolution, combined with the surface density of radio sources at around $1 \mathrm{Jy}$, does not cause a large fraction of confused sources, in some cases the probability that more than one object (especially those with flat radio spectra, such as blazars) falls within the WMAP $41 \mathrm{GHz}$ beam may not be negligible. When this happens, the microwave flux attributed to a WMAP foreground source may be contaminated. We analyzed all the WMAP beams centered on the point-like sources detected at $41 \mathrm{GHz}$ to determine the level of confusion produced by the presence of multiple radio sources in the same beam, taking into consideration the effect of primary beam attenuation (Page et al. 2003). For this purpose, we adopted the following procedure:

- we started our analysis by associating the WMAP foreground source with the radio source listed in the WMAP 3 year catalogue (Hinshaw et al. 2007). However, in some cases we found arguments to propose a different counterpart, as specifically discussed in Sect. 2.3 below;

- we choose to identify radio sources potentially contaminating the $41 \mathrm{GHz}$ flux to be all sources within the WMAP $41 \mathrm{GHz}$ beam centered on the object associated with the WMAP source, that have radio fluxes at $5 \mathrm{GHz}$ given by $f_{5 \mathrm{GHz}}>100 \mathrm{mJy}$;

- for each radio source detected at $5 \mathrm{GHz}$ within the WMAP beam, we estimated its $41 \mathrm{GHz}$ flux by extrapolating from its observed flux at lower frequencies using a linear regression (i.e., a power-law spectrum) technique. To this aim, we used a variety of (non-simultaneous) radio catalogues including SUMSS, NVSS, GB6, PMN, and CRATES. Finally, we corrected the central WMAP source flux by subtracting the $41 \mathrm{GHz}$ flux contributions of the possible contaminating objects by taking into account the distance-dependent primary beam attenuation of the WMAP $41 \mathrm{GHz}$ channel.

Since the minimum error in the WMAP flux is $0.1 \mathrm{Jy}$, the correction was applied only when it was larger than this value. Our method could suffer from variability of the contaminants, which are mostly flat-spectrum radio sources, but this is unlikely to be a major problem because of the small fraction of "corrected" sources and the level of contamination. The percentage of sources, that had their $41 \mathrm{GHz}$ flux corrected is only $\sim 7 \%$, the mean correction being $\sim 13 \%$.

An example of a WMAP source that is confused by another flatspectrum radio source in the field is shown in Fig. 1.

This field includes two flat spectrum radio sources in the WMAP 41-GHz channel beam (the red circle with radius $F W H M=31.8$ arcmin): the central source is located 1.8 arcsec from the center of the field and has a flat spectrum with slope $\alpha_{1.4-4.8 \mathrm{GHz}}=-0.05$; there is a second bright flat spectrum source at 15 arcmin from the center of the field.

Figure 2 shows a typical case in which there is no doubt about the association between the WMAP source and its radio counterpart. In this case, there is only one flat-spectrum $\left(\alpha_{1.4-4.8 \mathrm{GHz}}=0.1\right)$ radio source in the WMAP 41-GHz channel beam, and it is very close $(1.8 \mathrm{arcsec})$ to the center of the field.

\subsection{Object classification}

We follow standard criteria and classify our sources based on their optical and radio spectra. The blazar class includes BL Lacertae objects, historically characterized by an almost complete lack of emission lines, and FSRQs, which by definition 


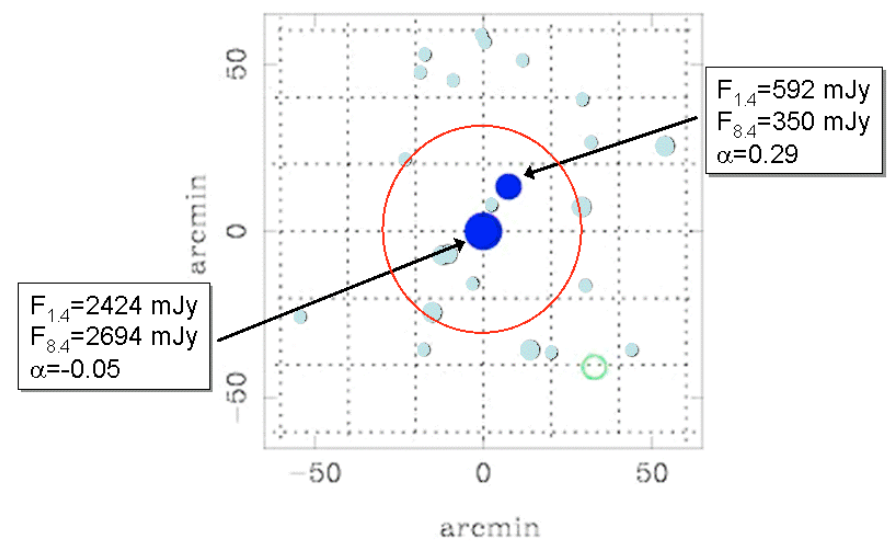

Fig. 1. An example of source confusion in the field of WMAP3 J03390144. Light grey filled circles represent radio sources (NVSS), either with steep radio spectrum or without spectral information; flat-spectrum radio sources are shown as dark blue filled circles. The green open circle marks the galaxy cluster ZW0334-0237, which is located 52 arcmin away from the center of the field. The size of the gray and blue circles is proportional to the $1.4 \mathrm{GHz}$ radio flux of the source they represent.

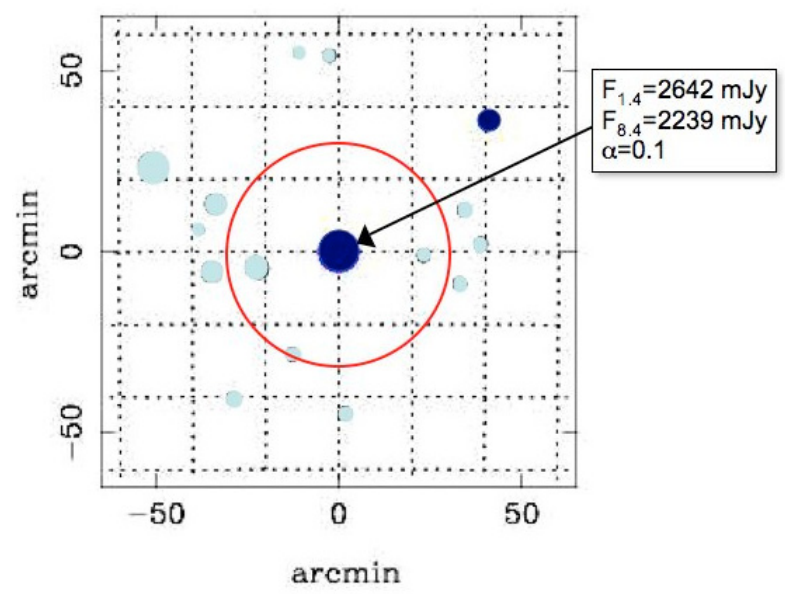

Fig. 2. Same as Fig. 1 but for the field of WMAP3 J2348-1631. The various sources in the field are NVSS sources either with steep radio spectrum or without spectral information (gray open circles) and flatspectrum radio sources (blue open circles). The size of the gray and blue circles is proportional to $1.4 \mathrm{GHz}$ radio flux. The WMAP source is clearly identified with the bright flat spectrum radio source (the blazar PKS 2345-16) at the center of the field.

display broad, strong emission lines. A dividing value of fullwidth half maximum $F W H M=1000 \mathrm{~km} \mathrm{~s}^{-1}$, between "narrow" and "broad" emission lines, is typically used. BL Lacs and radio galaxies can both have narrow lines, so their separation is typically determined on the basis of the value of the Ca break, a stellar absorption feature in the optical spectrum defined by $C=\left(f_{+}-f_{-}\right) / f_{+}$, where $f_{-}$and $f_{+}$are the fluxes in the restframe wavelength regions 3750-3950 $\AA$ and 4050-4250 $\AA$, respectively. A separation value of $C=0.4$ is normally adopted (e.g., Padovani et al. 2007), since sources with $C<0.4$ are believed to be dominated by non-stellar emission. We also make the commonly accepted distinction between steep spectrum radio quasars (SSRQ), $\alpha_{\mathrm{r}}>0.5$, and FSRQ, $\alpha_{\mathrm{r}} \leq 0.5$. To separate BL Lacs (with broad emission lines) from radio quasars, a limit of $5 \AA$ to the rest-frame equivalent width of any detected emission line is normally used (e.g., Stickel et al. 1991).
Since most of the objects in the WMAP 3-year catalogue are bright and well known radio sources, the literature is rich with data. We used the broad-band SED as well as any optical spectroscopy information available in the literature (mostly taken from the blazar catalog of Massaro et al. 2009, from the NASA Extragalactic Database and from the SDSS-DR6 on-line services) to classify all sources in the WMAP 3-year catalogue.

In summary, a WMAP source is associated with:

- an FSRQ, if its spectral index at $\mathrm{cm}$ frequencies $\alpha_{\mathrm{r}}$ between the radio and the microwave region $\alpha_{1.4-41 \mathrm{GHz}}$ is flatter than 0.5 , and if its optical spectrum shows strong and broad emission lines typical of QSOs, as described above;

- a BL Lac, if its spectral index at $\mathrm{cm}$ frequencies $\alpha_{\mathrm{r}}$ between the radio and the microwave region $\alpha_{1.4-41 \mathrm{GHz}}$ is flatter than 0.5, and if its optical spectrum does not show strong broad emission lines and looks non-thermal, as detailed above;

- an SSRQ, if its spectral index at $\mathrm{cm}$ frequencies $\alpha_{\mathrm{r}}$ is steeper than 0.5 (independently of the $\alpha_{1.4-41 \mathrm{GHz}}$ index, which could be significantly flatter than $\alpha_{\mathrm{r}}$ ) and if its optical spectrum shows strong and broad emission lines;

- a radio galaxy, if its spectral index at $\mathrm{cm}$ frequencies $\alpha_{\mathrm{r}}$ is steeper than 0.5 (independently of the $\alpha_{1.4-41 \mathrm{GHz}}$ index), its radio emission is clearly extended, it has weak, narrow lines and an optical spectrum dominated by stellar emission, and if the host galaxy is clearly visible at optical/IR frequencies.

In a few cases, we could not find sufficient information to classify a source with blazar characteristics as an object belonging to one of the above categories. In the following, these sources are labelled as Blazars - Unknown type. Finally, in three cases the WMAP source appears to be classified in the literature as a starburst galaxy.

High frequency surveys detect a population of gigaHertz peaked spectrum (GPS) sources (e.g., O'Dea 1998). We crosscorrelated the WMAP list with the compilation by Labiano et al. (2007) and found 11 GPS sources, 5 of which belong to the complete sample. These objects are labelled accordingly in Table 3.

\subsection{Notes on individual objects}

WMAP3 J0026-3511 (complete sample). There are no optical spectra of this object available in the literature. Its very flat radio spectrum and its blazar-like SED make this source a highconfidence blazar candidate. This source is very weak at low frequencies with a flux of only $14 \mathrm{mJy}$ at $843 \mathrm{MHz}$ (from SUMSS) and $25 \mathrm{mJy}$ at $1.4 \mathrm{GHz}$ (from NVSS). It is listed in the CRATES catalogue (Healey et al. 2007) as a very inverted radio source (spectral slope $\alpha_{\mathrm{r}}=-1.4$ ) with a flux of 121 and $314 \mathrm{mJy}$, at 4.8 and $8.4 \mathrm{GHz}$, respectively.

WMAP3 J0210-5100 (complete sample). This source is a transition object between FSRQs and BL Lacs (Massaro et al. 2009). Given the relatively high redshift of this object $(z=1)$, we include it in the FSRQ sample.

WMAP3 J0540-5416, J0550-5732, J0633-2217, J1038+0511, J1102-4400, J1333+2723, J2035-6845 and J2148-7757 (complete sample: 1st, 2nd, and 4th source). These are all FSRQs that have were identified by Healey et al. 2008 as part of the identification program of the CGRaBS gamma-ray source candidates. 
WMAP3 J1149-7932. The object associated with this source in the WMAP 3-year catalog is not the most likely counterpart, since it is too faint and has a rather steep spectrum.

WMAP3 J1227+1124. The position of the WMAP source does not correspond to a definite radio source. The GB6 source in the field is rather faint (34 mJy) and has a flat spectrum. However, a bright radio source is associated to an interactive radio-galaxy (VPCX 27$) \approx 25$ arcmin away from the nominal position of the WMAP 3-year source.

WMAP3 J1231+1351. Hinshaw et al. (2007) associate this source with GB6 J1231+1344 which is located 10 arcmin away from the WMAP source centroid. However, this radio source has a steep radio spectrum, while the source GB6 J1232+1359, at about 20 arcmin from the WMAP centroid, is flat and brighter then GB6 J1231+1344. For this reason, we associate WMAP3 J1231+1351 with GB6 J1232+1359.

WMAP3 J1305-4928. The WMAP source is associated with the galaxy NGC 4945, one of the four brightest FIR sources in the sky outside the Local Group. NGC 4945 hosts both vigorous nuclear star formation (Moorwood \& Oliva 1994; Spoon et al. 2000) and a peculiar broad-lined AGN with an estimated bolometric luminosity of $\sim 60 \%$ of the nuclear FIR luminosity. We classified this object as FIR-starburst galaxy in our sample.

WMAP3 J1657+4749. We found 2 objects located inside the WMAP $41 \mathrm{GHz}$ beam: 4C 48.41 and [HB89] 1656+477, both with a flat radio spectrum. The WMAP source has a $41 \mathrm{GHz}$ flux of $0.6 \mathrm{Jy}$, but the two sources mentioned above exhibit predicted fluxes at $41 \mathrm{GHz}$ of $0.5 \mathrm{Jy}$ and $1.3 \mathrm{Jy}$, respectively. Hinshaw et al. (2007) associated this source with the object GB6 1658+4737, that is [HB89] 1656+477, and we agree with this preliminary association.

WMAP3 J1924-2914 (complete sample). Despite this source (PKS J1924-2914, PMN J1924-2914 or OV-236) being one of the brightest extragalactic objects at $\mathrm{mm}$ frequencies, it was not included in the WMAP 3-year catalog of Hinshaw et al. (2007) because it falls within the Kp0 cut that is used to avoid including a large number of Galactic sources in the catalog. Since the Kp0 cut is based on the WMAP $K$-band intensity and this source is so bright, it falls within that cut even though it is a relatively high latitude source $\left(b_{\mathrm{II}}=-19.6^{\circ}\right)$; this is the only high-latitude extragalactic source to suffer this fate (Hinshaw private communication; see also Fig. 2 of Bennett et al. 2003). PKS J1924-2914, a well known, bright FSRQ, was clearly detected in the WMAP maps in all bands. Therefore, we included it in our blazar sample. We estimated (from an analysis of the WMAP 3-yr data) that this source has a flux of $f_{41 \mathrm{GHz}}=13.29 \pm 1.12 \mathrm{Jy}$ (with a $\mathrm{mm}$ spectral index $\alpha=0.6$ ) and so it has been included in our complete sample.

WMAP3 J2333-2340 (complete sample). The optical spectrum of this source (Wilkes et al. 1983) shows emission lines and therefore, for consistency with current literature classification methods, we classify it as a radio galaxy. However the SED is typical of a blazar at all frequencies and shows a large optical variability. In a high optical state, this object would have been classified as a BL Lac.

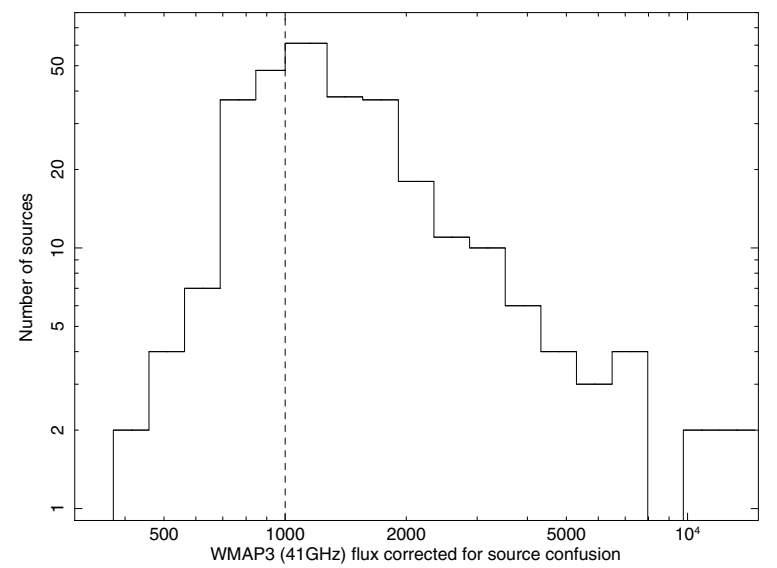

Fig. 3. The distribution of the corrected $41 \mathrm{GHz}$ flux in the entire WMAP sample. The sample appears to be clearly incomplete below $1 \mathrm{Jy}$, as indicated by the dashed vertical line.

\subsection{The complete sample}

The full list of WMAP sources is given in Table 3, where Col. 1 gives the WMAP source name following the IAU naming convention, Col. 2 gives the WMAP number defined in the first year catalogue (if available), and Col. 3 gives a common name (e.g. NGC, PKS, 3C etc.) if the source is a previously known object; Cols. 4 and 5 give the right ascension and declination (J2000.0) of the counterpart of the WMAP source; Col. 6 gives the source class following the classification scheme described in Sect. 2.2; Col. 7 gives the redshift when available; Cols. 8 and 9 give the flux at $5 \mathrm{GHz}$ (from the literature) and at $41 \mathrm{GHz}$ (from the WMAP-3 yr catalogue); Col. 10 gives the $41 \mathrm{GHz}$ flux corrected for source confusion as described in Sect. 2.1, and Col. 11 states whether or not the source is part of the flux-limited, complete sample.

The flux list of WMAP sources and the limited sample that we use for our statistical analysis is defined as the sample of all sources in the WMAP 3-yr catalogue with fluxes at $41 \mathrm{GHz}$ higher than $1 \mathrm{Jy}$ and with Galactic latitude $\left|b_{\mathrm{II}}\right|>15^{\circ}$. The latter condition is necessary to limit source confusion and the complications associated with the emission close the Galactic plane. Taking also into account the higher latitude regions excluded by the Kp0 mask of (Bennett et al. 2003) our total area is 28457 square degrees. We chose $f_{41 \mathrm{GHz}}=1 \mathrm{Jy}$ as the flux limit of our sample because the number of sources below this flux value drops sharply as shown in Fig. 3, where the distribution of $41 \mathrm{GHz}$ fluxes is plotted for the entire sample. Above $F_{41 \mathrm{GHz}}=1 \mathrm{Jy}$ (marked by the vertical dashed line), the slope of the distribution is steep and uniform indicating a very good level of completeness even close to the flux limit.

Table 1 provides the statistics of the WMAP source identification both for the full and the complete sample.

\section{The blazar number counts at microwave frequencies $(41 \mathrm{GHz})$}

We present the $41 \mathrm{GHz} \log N-\log S$ of blazars derived from our complete sample and compare it with the counts derived at radio $(5 \mathrm{GHz})$ frequency. Figure 4 shows the integral $\log N-\operatorname{logS}$ of all blazars (i.e., FSRQs, BL Lacs, and unclassified types) in the complete sample. The blazar counts at $41 \mathrm{GHz}$ are steep and described well by a simple power law of the type $N(>S) \propto S^{-\beta}$. We use the maximum likelihood method (Crawford et al. 1970) 
Table 1. Summary of WMAP objects identification.

\begin{tabular}{lc}
\hline \hline Source type & $\begin{array}{c}\text { Number in sample } \\
\text { total/complete }\end{array}$ \\
\hline FSRQ & $202 / 137$ \\
BL Lac & $38 / 24$ \\
Blazars-unknown type & $17 / 11$ \\
SSRQ & $14 / 9$ \\
Radio galaxies & $31 / 15$ \\
Starburst galaxies & $3 / 2$ \\
Planetary/LBN nebulae & $4 / 2$ \\
Unidentified/no radio-counterpart & $15 / 3$ \\
Total & $324 / 203$ \\
\hline
\end{tabular}

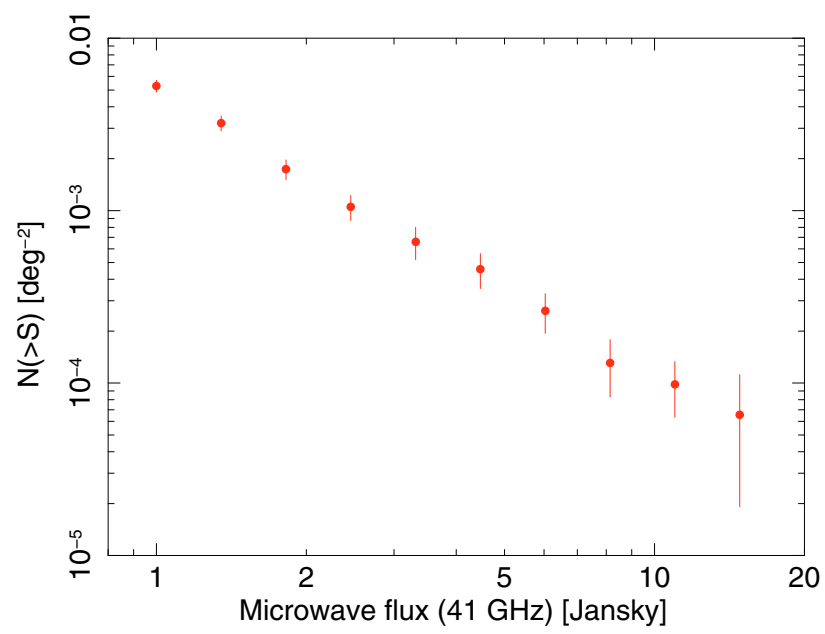

Fig. 4. The $41 \mathrm{GHz} \log N-\log S$ of all blazars in the complete sample $\left(\left|b_{\mathrm{II}}\right|>15^{\circ}\right)$ is shown by the filled dots with error bars. For clarity, only a subset of points is shown.

to estimate the slope of the number counts using all available data $^{2}$. This method yields $\beta=1.64 \pm 0.14$, somewhat steeper than the Euclidean value of 1.5. The slopes for blazar subclasses and other classes are consistent with this value within the typically large error bars.

This $41 \mathrm{GHz} \log N-\log S$ is in very good agreement in terms of both slope and normalization with the $5 \mathrm{GHz}$ blazar counts at flux densities $>1$ Jy (see e.g., Giommi et al. 2006). This is expected given the flat $\left\langle\alpha_{5-41 \mathrm{GHz}}\right\rangle=0.02$ average spectral slope of our blazars.

\section{Cosmological evolution}

The simplest way to study the evolutionary properties of a sample is by means of the $V / V_{\max }$ test (Schmidt 1968). Values of $\left\langle V / V_{\max }\right\rangle$ that differ significantly from 0.5 indicate evolution, which is either positive (i.e., sources were more luminous and/or more numerous in the past) for values $>0.5$, or negative (i.e., sources were less luminous and/or less numerous in the past) for values $<0.5$. One can also fit an evolutionary model to the sample by finding the evolutionary parameter that reproduces $\left\langle V / V_{\max }\right\rangle=0.5$.

We computed the quantity $V / V_{\max }$ for our sources, with statistical errors given by $\sigma=1 / \sqrt{12 N}$ (Avni \& Bahcall 1980). To derive a simple estimate of the sample evolution, we also derived the best-fit model parameter $\tau$ (given in units of the age of

2 This method operates on the differential distribution and obviates to the fact that individual points in integral counts are not independent.

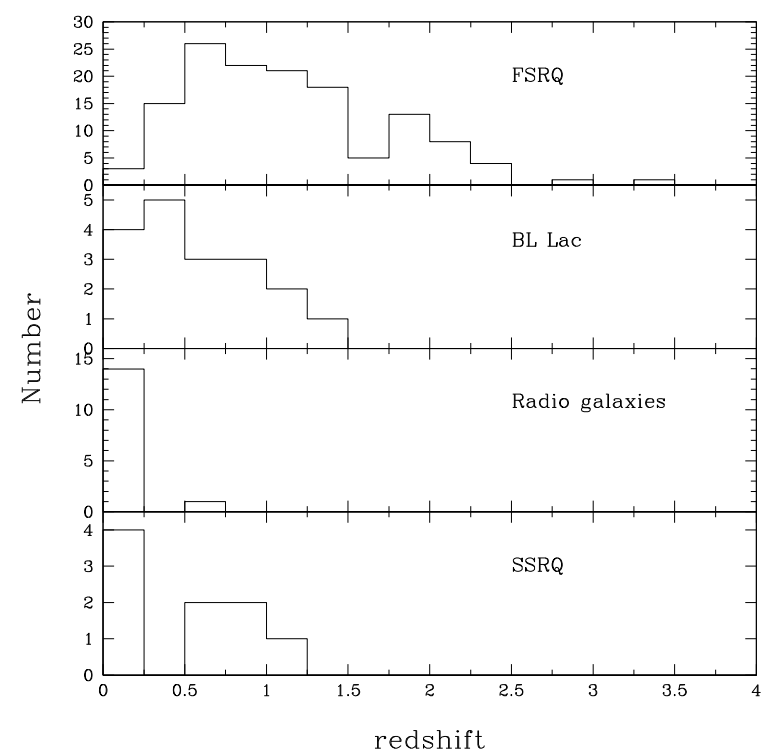

Fig. 5. The redshift distributions of blazars (FSRQs and BL Lacs), SSRQs, and radio galaxies.

the universe) of a pure luminosity evolution model, i.e., $P(z)=$ $P(0) \exp [T(z) / \tau]$, where $T(z)$ is the adimensional lookback time: the smaller $\tau$ is the stronger the evolution. We assume here that some luminosity evolution takes place, based on previous studies in the radio and other bands (e.g., Padovani et al. 2007; Croom et al. 2004).

The redshift distribution of WMAP blazars, SSRQs, radio galaxies is shown in Fig. 5.

The results of the cosmological evolution for our sample are shown in Table 2, which lists the source subsample in Col. (1), the number of sources in Col. (2), the mean redshift in Col. (3), the value of $\left\langle V / V_{\max }\right\rangle$ and $\tau$ in Cols. (4) and (5) for our $\Lambda \mathrm{CDM}$ reference cosmological model, and the value of $\left\langle V / V_{\max }\right\rangle$ and $\tau$ in Cols. (6) and (7) for an empty universe cosmology, for comparison with previous results.

The main results of our analysis can be summarized as follows:

1. FSRQs evolve at the $4.6 \sigma$ level $\left(\left\langle V / V_{\max }\right\rangle=0.614 \pm 0.025\right)$, confirming a well known result (e.g., Padovani \& Urry 1992; Urry \& Padovani 1995; Padovani et al. 2007). The evolutionary parameter of this class is consistent with that derived by (Urry \& Padovani 1995) for the 2 Jy FSRQ sample in an empty universe $\left(\tau=0.23_{-0.04}^{+0.07}\right)$, for the simple case of pure luminosity evolution.

2. BL Lacs display moderate evolution, i.e., their value of $\left\langle V / V_{\max }\right\rangle=0.62 \pm 0.06$ is different from 0.5 at the $2 \sigma$ level. That $25 \%$ of the sources have no redshift information is not a significant problem, since redshift evolution affects $V / V_{\max }$ values far less than if affects flux.

3 . The properties of non-blazars are consistent with experiencing no evolution (radio galaxies $\left\langle V / V_{\max }\right\rangle=0.61 \pm 0.07$ and $\left.\operatorname{SSRQ}\left\langle V / V_{\max }\right\rangle=0.63 \pm 0.10\right)$. However, this is probably due to small number statistics, since SSRQ and high-power radio galaxies are known to evolve strongly in the radio band (e.g., Urry \& Padovani 1995).

4. Blazars of unknown type show hints of evolution $\left(\left\langle V / V_{\max }\right\rangle=0.70 \pm 0.09\right)$ at the $2.3 \sigma(2.5 \sigma$ for an empty universe cosmology) level, with a best-fit model value of $\tau$ being consistent, within $\sim 1.5 \sigma$, with that of FSRQs. 
Table 2. WMAP sample evolutionary properties.

\begin{tabular}{|c|c|c|c|c|c|c|}
\hline Class & Number & $\langle z\rangle$ & $\begin{array}{l}H_{0}=70, \Omega_{\mathrm{M}}=0 \\
\left\langle V / V_{\max }\right\rangle\end{array}$ & $\begin{array}{c}\Omega_{\Lambda}=0.7 \\
\tau\end{array}$ & $\begin{array}{l}H_{0}=50, \Omega_{\mathrm{M}}= \\
\left\langle V / V_{\max }\right\rangle\end{array}$ & $\begin{array}{c}\overline{0, \Omega_{\Lambda}}=0 \\
\tau\end{array}$ \\
\hline FSRQ & 137 & $1.13 \pm 0.05$ & $0.614 \pm 0.025$ & $0.35_{-0.05}^{+0.07}$ & $0.621 \pm 0.025$ & $0.26_{-0.03}^{+0.05}$ \\
\hline BL Lacs & 24 & $0.56 \pm 0.09^{a}$ & $0.619 \pm 0.059^{b}$ & $0.31_{-0.21}^{+0.21}$ & $0.626 \pm 0.059^{b}$ & $0.24_{-0.05}^{+0.15}$ \\
\hline Radio Galaxies & 15 & $0.08 \pm 0.04$ & $0.610 \pm 0.074$ & $\ldots^{-c .08}$ & $0.610 \pm 0.074$ & $\ldots{ }^{-0.03}$ \\
\hline Unclassified blazars & 11 & $0.77 \pm 0.16^{d}$ & $0.703 \pm 0.087^{e}$ & $0.22^{+0.11}$ & $0.713 \pm 0.087^{e}$ & $0.17^{+0.07}$ \\
\hline SSRQ & 9 & $0.50 \pm 0.14$ & $0.626 \pm 0.096$ & $\ldots^{-c^{0.04}}$ & $0.632 \pm 0.096$ & $\ldots^{-c^{-0.03}}$ \\
\hline
\end{tabular}

${ }^{a}$ Excluding the 6 sources without redshift; ${ }^{b}$ assuming $z=\langle z\rangle$ for the sources without redshift; ${ }^{c}\left\langle V / V_{\max }\right\rangle$ not significantly different $(<2 \sigma)$ from 0.5 : no evolution assumed; ${ }^{d}$ excluding the 6 sources without redshift; ${ }^{e}$ assuming $z$ equal to the mean blazar redshift for the sources without redshift.

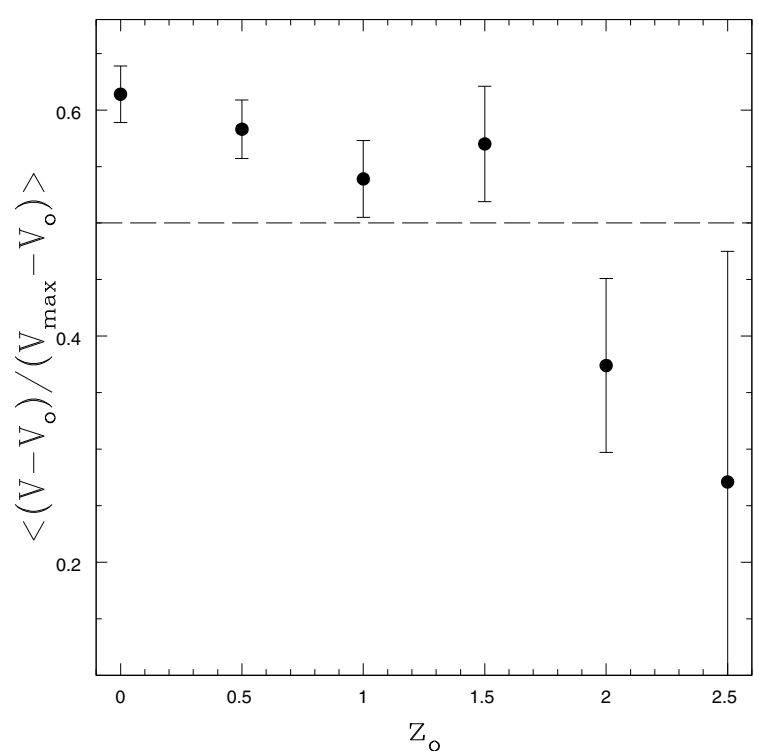

Fig. 6. The banded statistic, $\left\langle\left(V-V_{\mathrm{o}}\right) /\left(V_{\max }-V_{\mathrm{o}}\right)\right\rangle$ versus $z_{\mathrm{o}}$ for WMAP FSRQ. The horizontal dashed line indicates the value of 0.5 expected based on the null hypothesis of no evolution.

Given the available FSRQ statistics, we can move beyond the assumption of pure luminosity evolution and study possible redshift dependencies. We do this by using the so-called banded $\left\langle V / V_{\max }\right\rangle$ statistic, i.e., the quantity $\left\langle\left(V-V_{\mathrm{o}}\right) /\left(V_{\max }-V_{\mathrm{o}}\right)\right\rangle$, where $V_{\mathrm{o}}$ is the cosmological volume enclosed by a given redshift $z_{\mathrm{o}}$ (Dunlop \& Peacock 1990). This procedure allows the detection of any high redshift (possibly negative) evolution by separating it from the well-known strong (positive) low redshift evolution. Figure 6 shows that $\left\langle\left(V-V_{\mathrm{o}}\right) /\left(V_{\max }-V_{\mathrm{o}}\right)\right\rangle$ is basically constant up to $z \sim 1.5$ with a significant drop at higher redshifts. Its value decreases from being $>0.5$ at the $4.6 \sigma$ level at $z \sim 0$, to values $\leq 0.5$ for $z \gtrsim 2$. However, given that only $\sim 10 \%$ of the FSRQ are above this redshift, we assume a pure luminosity evolution for the whole sample. This is confirmed, within the somewhat limited statistics, by a study of the LF in redshift bins.

\section{The luminosity function of WMAP blazars}

\subsection{BL Lacs}

The local LF of WMAP BL Lacs is shown in Fig. 7. Based on the $\left\langle V / V_{\max }\right\rangle$ analysis, this was de-evolved to zero redshift using $\tau=0.31$. We assumed that $z=\langle z\rangle \sim 0.56$ for the $6 / 24$ BL Lacs without a redshift.

We fitted the LF with a single power law $\phi\left(P_{\mathrm{r}}\right) \propto P_{\mathrm{r}}^{-B_{\mathrm{r}}}$. Varying the luminosity binning, the differential slope is in the range $2.4<B_{\mathrm{r}}<2.7$. For a representative bin size of

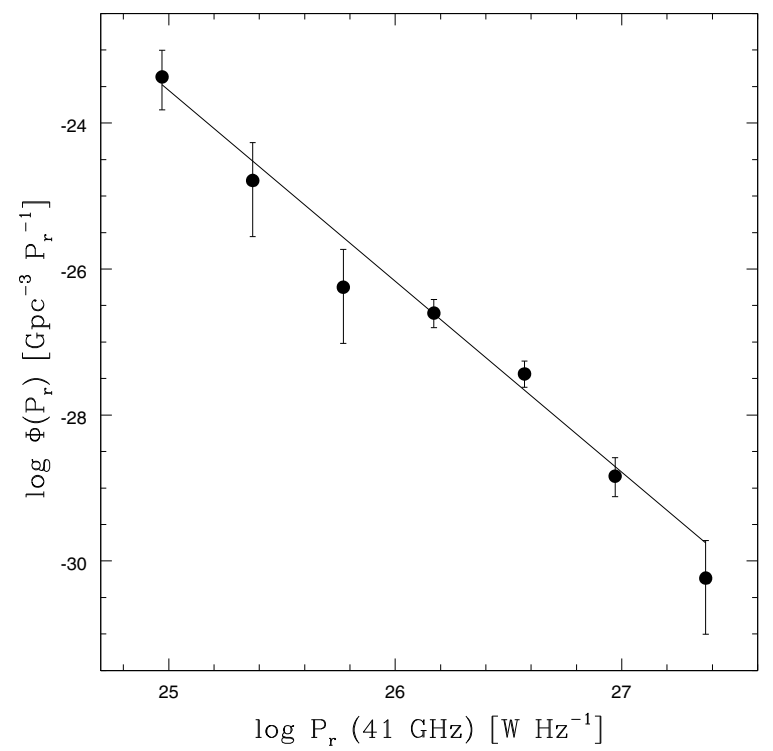

Fig. 7. The local, de-evolved $41 \mathrm{GHz}$ luminosity function of BL Lacs (filled points with error bars). Error bars correspond to $1 \sigma$ Poisson errors (Gehrels 1986). The solid line is a weighted least squares fit to the data of the form $\phi\left(P_{\mathrm{r}}\right) \propto P_{\mathrm{r}}^{-2.62}$ (see text for details).

$\Delta \log P_{\mathrm{r}}=0.4$, a weighted least squares fit yields $\phi\left(P_{\mathrm{r}}\right) \propto$ $P_{\mathrm{r}}^{-2.62 \pm 0.19}\left(\chi_{v}^{2} \sim 0.94\right.$ for 5 degrees of freedom). The total number density of BL Lacs in the luminosity range $7 \times$ $10^{24}-10^{28} \mathrm{~W} / \mathrm{Hz}$, derived independently of bin size from the integral LF, which is equal to $\sum 1 / V_{\max }$, is $44 \pm 29 \mathrm{Gpc}^{-3}$ (see Eqs. (9) and (10) of Condon et al. 2002).

We note that our assumption about missing redshifts could somewhat bias our LF. Therefore, we checked this by assuming that $z=1$ for these sources. As discussed in Padovani et al. (2007), their featureless continuum might indeed be indicative of a relatively high redshift. However, this assumption produces a LF that is consistent with the previous one well within the margins of the errors, with $\phi\left(P_{\mathrm{r}}\right) \propto P_{\mathrm{r}}^{-2.51 \pm 0.19}$ $\left(\chi_{v}^{2} \sim 0.69\right.$ for 5 degrees of freedom). We have also derived the bivariate LF of WMAP BL Lacs at $5 \mathrm{GHz}$ for an empty universe cosmology, to compare it with previous determinations and with the predictions of unified schemes. This is presented in Fig. 8 (filled points), which also shows the DXRBS (open triangles, Padovani et al. 2007), 1 Jy LF (open squares, Stickel et al. 1991), and the predictions of a beaming model based on the 1 Jy LFs and evolution (solid line, Urry \& Padovani 1995). These predictions assume that Fanaroff-Riley type I (FR I; Fanaroff \& Riley 1974) galaxies are the counterparts of BL Lacs with their jets being in the plane of the sky, and show what one should expect to find when reaching powers lower than those used to constrain the LF at the high 


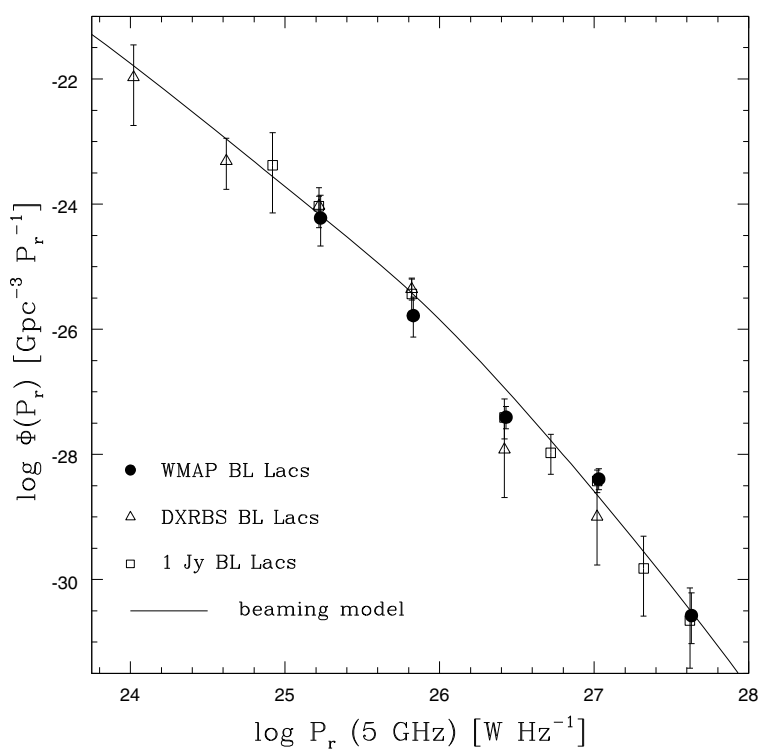

Fig. 8. The bivariate $5 \mathrm{GHz}$ luminosity function of WMAP BL Lacs (filled points) and DXRBS (open triangles) compared to the predictions of a beaming model based on the 1 Jy luminosity function and evolution (solid line Urry \& Padovani 1995). The open squares represent the 1 Jy luminosity function (Stickel et al. 1991). Error bars correspond to $1 \sigma$ Poisson errors (Gehrels 1986). The DXRBS points were corrected to take into account their somewhat different definition of a BL Lac (Padovani et al. 2007). For consistency with (Stickel et al. 1991) and (Urry \& Padovani 1995), the WMAP luminosity function was deevolved to zero redshift, and an $H_{0}=50 \mathrm{~km} \mathrm{~s}^{-1} \mathrm{Mpc}^{-1}, \Omega_{\mathrm{M}}=0$, and $\Omega_{\Lambda}=0$ cosmology has been adopted.

end. For consistency with (Stickel et al. 1991) we de-evolved the WMAP BL Lacs assuming $\tau=0.32$, which is within $0.5 \sigma$ from our own value (see Table 2). We note that $14 / 34$ of the $1 \mathrm{Jy}$ BL Lacs also belong to the WMAP sample.

The LF of WMAP BL Lacs is in very good agreement, in the region of overlap, with the DXRBS and 1 Jy LFs. In particular, the WMAP and 1 Jy LFs cover roughly the same power range, a result that is expected given the relatively similar flux limits. The WMAP LF is also in good agreement with the predictions of unified schemes. For this cosmology, the total number density of BL Lacs in the luminosity range $2 \times 10^{25}-4 \times 10^{27} \mathrm{~W} / \mathrm{Hz}$, derived independently of bin size from the integral LF, is $17 \pm$ $11 \mathrm{Gpc}^{-3}$ to be compared with the value of $40 \mathrm{Gpc}^{-3}$ in the range $6 \times 10^{24}-3 \times 10^{27} \mathrm{~W} / \mathrm{Hz}$ for the $1 \mathrm{Jy} \mathrm{LF}$.

\subsection{FSRQs}

The local LF of WMAP FSRQs is shown in Fig. 9. Based on the $\left\langle V / V_{\max }\right\rangle$ analysis, this was de-evolved to zero redshift using $\tau=0.35$.

We fitted the LF with a single power law $\phi\left(P_{\mathrm{r}}\right) \propto P_{\mathrm{r}}^{-B_{\mathrm{r}}}$. Varying the binning, the differential slope is in the range $2.1<$ $B_{\mathrm{r}}<2.5$. For a bin size $\Delta \log P_{\mathrm{r}}=0.4$, which is representative, a weighted least squares fit yields $\phi\left(P_{\mathrm{r}}\right) \propto P_{\mathrm{r}}^{-2.3 \pm 0.1}\left(\chi_{v}^{2} \sim 5.1\right.$ for 6 degrees of freedom). A single power law cannot then reproduce the observed LF (see Fig. 9). We note however that the first low-power bin includes both a single source (3C 120) and the somewhat large gap between the first and the second bin. We note that other authors have classified 3C 120 as a broad line radio galaxy.

The total number density of FSRQs in the luminosity range $6 \times 10^{24}-10^{28} \mathrm{~W} / \mathrm{Hz}$, derived independently of bin size from the

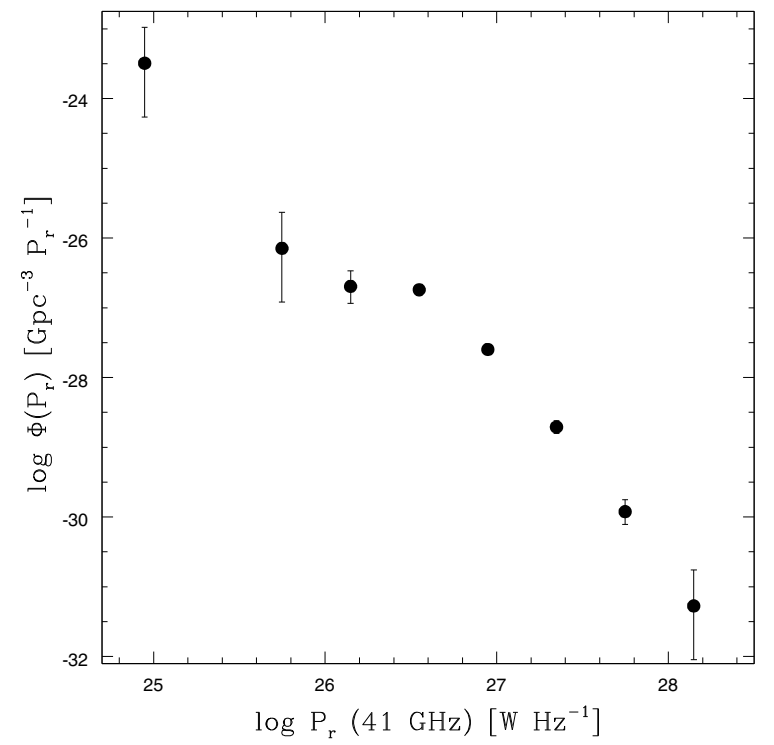

Fig. 9. The local, de-evolved $41 \mathrm{GHz}$ luminosity function of WMAP FSRQs. Error bars correspond to $1 \sigma$ Poisson errors (Gehrels 1986).

integral LF, is $30 \pm 28 \mathrm{Gpc}^{-3}$. Excluding the lowest luminosity source, one obtains $1.6 \pm 0.4 \mathrm{Gpc}^{-3}$ in the luminosity range $7 \times$ $10^{25}-10^{28} \mathrm{~W} / \mathrm{Hz}$.

As already done for BL Lacs, we also derived the local bivariate LF of FSRQs at $5 \mathrm{GHz}$ for an empty universe cosmology, to compare it with previous determinations and with the predictions of unified schemes. Based on the $\left\langle V / V_{\max }\right\rangle$ analysis, this bivariate LF was de-evolved using the value $\tau=0.26$. The bivariate LF is show in Fig. 10 (filled points), which also shows the DXRBS (open triangles, Padovani et al. 2007) and 1 Jy LFs (open squares, Stickel et al. 1991), and the predictions of a beaming model based on the $2 \mathrm{Jy} \mathrm{LF}$ and evolution (solid line, Urry \& Padovani 1995). Given that the DXBRS evolutionary parameter is epoch dependent and that therefore we cannot simply de-evolve the global LF to zero redshift, we restricted ourselves to sources with $z \leq 1$ (see Padovani et al. 2007 for details). We note that $36 / 52$ of the 2 Jy and $8 / 129$ of the DXRBS FSRQ belong to the WMAP sample.

The WMAP LF is in very good agreement, in the region of overlap, with the DXRBS and 2 Jy LFs. In particular, the WMAP and 2 Jy LFs cover roughly the same power range, as expected given the relatively similar flux limits. The WMAP LF is also in relatively good agreement with the predictions of unified schemes. Namely, most bins agree to $1-2 \sigma$ with the beaming model, the only exception being the two bins around $10^{27} \mathrm{~W} / \mathrm{Hz}$.

\subsection{Radio galaxies and SSRQs}

The LF of WMAP radio galaxies is shown in Fig. 11. Based on the $\left\langle V / V_{\max }\right\rangle$ analysis, no evolution was assumed. We fitted the LF with a single power law $\phi\left(P_{\mathrm{r}}\right) \propto P_{\mathrm{r}}^{-B_{\mathrm{r}}}$. Varying the binning, the differential slope was found to be in the range $2.4<B_{\mathrm{r}}<2.7$. For a representative bin size $\Delta \log P_{\mathrm{r}}=0.5$, a weighted least squares fit yields $\phi\left(P_{\mathrm{r}}\right) \propto P_{\mathrm{r}}^{-2.6 \pm 0.1}\left(\chi_{v}^{2} \sim 0.17\right.$ for 5 degrees of freedom). The total number density of radio galaxies in the luminosity range $2 \times 10^{23}-10^{27} \mathrm{~W} / \mathrm{Hz}$, which was derived independently of bin size from the integral LF, is $10500 \pm 5400 \mathrm{Gpc}^{-3}$.

As for SSRQs, their sample is too small to derive a meaningful LF. However, we can say that their total number density in the 


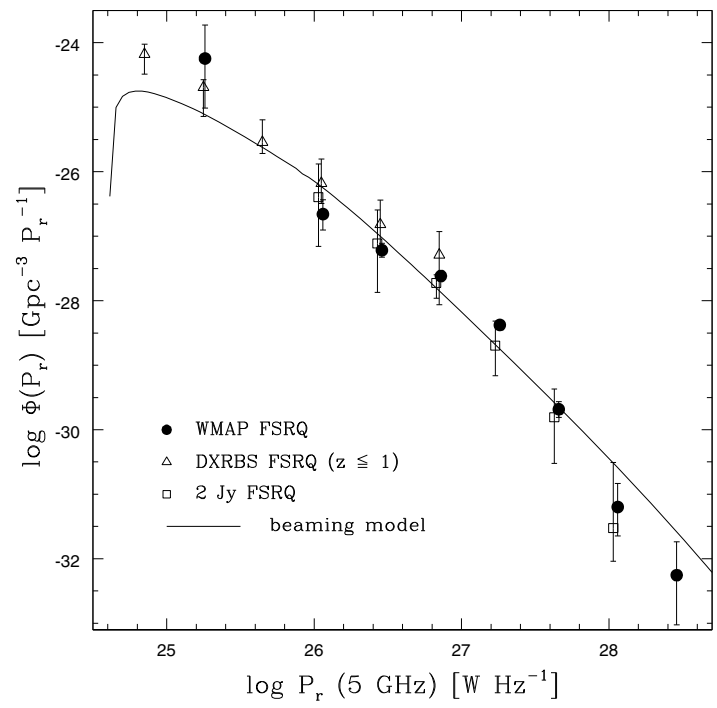

Fig. 10. The local, de-evolved bivariate $5 \mathrm{GHz}$ radio luminosity function of WMAP FSRQs (filled points) and DXRBS with $z \leq 1$ (open triangles) compared to the predictions of a beaming model based on the $2 \mathrm{Jy}$ luminosity function and evolution (solid line Urry \& Padovani 1995). The open squares represent the 2 Jy luminosity function. Error bars represent the sum in quadrature of the $1 \sigma$ Poisson errors (Gehrels 1986) and the variations of the number density associated with a $1 \sigma$ change in the evolutionary parameter $\tau$. For consistency with Urry \& Padovani (1995) an empty cosmology with $H_{0}=50 \mathrm{~km} \mathrm{~s}^{-1} \mathrm{Mpc}^{-1}, \Omega_{\mathrm{M}}=0$, and $\Omega_{\Lambda}=0$ has been adopted. Both beaming model and 2 Jy LF have been converted from $2.7 \mathrm{GHz}$ assuming $\alpha_{\mathrm{r}}=0$.

luminosity range $5 \times 10^{24}-4 \times 10^{27} \mathrm{~W} / \mathrm{Hz}$, derived independently of bin size from the integral LF, is $91 \pm 59 \mathrm{Gpc}^{-3}$.

\section{Discussion and conclusions}

We have presented a detailed study of the counterparts of all sources of the WMAP 3-year catalogue, which has led to the identification of 309 microwave-selected objects. We have found that only 7 sources (three planetary nebulae, one Lynds Bright Nebula, and three starburst galaxies) are neither blazars nor non-thermal misdirected AGN (i.e., radio galaxies and steepspectrum radio quasars). All the remaining 302 sources have been classified according to the scheme presented in Sect. 2.2. This result fully confirms the previous findings of Giommi \& Colafrancesco (2004) that the vast majority of WMAP-detected sources are blazars or radio galaxies.

After correcting their fluxes for source confusion within the WMAP $41 \mathrm{GHz}$ channel beam, we have used the WMAP 3year source catalogue to define a microwave flux-limited, virtually complete, sample of blazars with $F_{41 \mathrm{GHz}}>1 \mathrm{Jy}$. Only 3 of the 203 objects in this sample remain unidentified. This is the first statistically clearly defined and complete sample of blazars selected at microwave frequencies. The microwave frequency range is probably the optimal region of the entire electromagnetic spectrum to pursue statistical studies of blazars since it is least affected by the superposition of spectral components of different nature, such as steep radio emission from the extended part of the jet, non-nuclear optical emission from the host galaxy or optical/UV, and soft X-ray emission from an accretion disk.

Our complete sample allows us to derive the number counts, luminosity function, and cosmological evolution of WMAP AGN. We found that the $\log N-\log S$ of microwave-selected

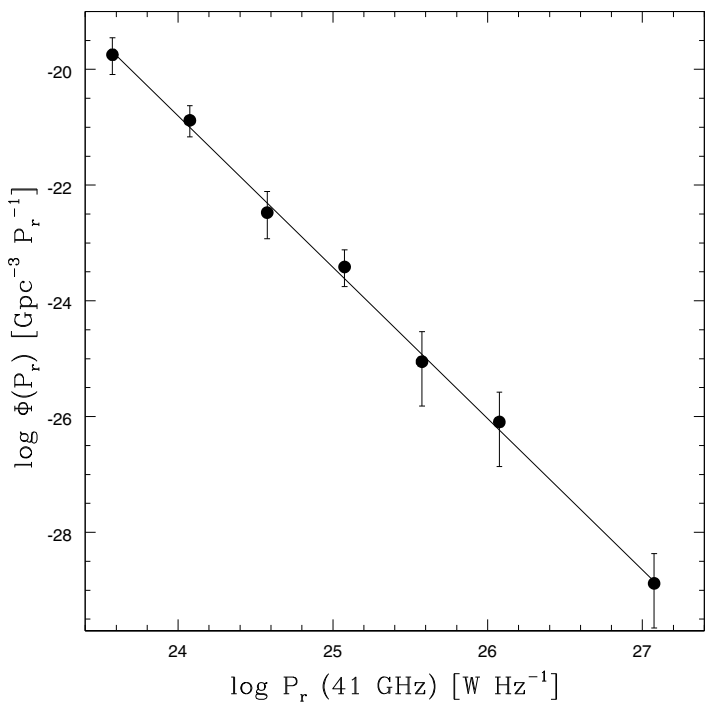

Fig. 11. The $41 \mathrm{GHz}$ luminosity function of radio galaxies (filled points). Error bars correspond to $1 \sigma$ Poisson errors (Gehrels 1986). The solid line is a weighted least squares fit to the data of the form $\phi\left(P_{\mathrm{r}}\right) \propto P_{\mathrm{r}}^{-2.6}$ (see text for details).

blazars is steep, $N(>S) \sim S^{-1.64}$, down to $1 \mathrm{Jy}$, consistently with that found at $\mathrm{cm}$ frequencies (Giommi \& Colafrancesco 2004, 2005).

The bivariate $5 \mathrm{GHz}$ luminosity functions of FSRQs and BL Lacs are similar to those derived from DXRBS (for FSRQs and BL Lacs Padovani et al. 2007) and from 2-Jy and 1Jy FSRQs and BL Lacs surveys, respectively. A beaming model (Urry \& Padovani 1995) adapted to the 1-Jy and 2-Jy blazar surveys is able to reproduce the observed luminosity function of WMAP blazars down to $P_{\mathrm{r}}(5 \mathrm{GHz}) \sim 10^{25} \mathrm{~W} / \mathrm{Hz}$.

The cosmological evolution of these two subclasses of AGN is consistent with the expectations based on radio/cm measurements, yielding evolution at the $2 \sigma$ level for BL Lacs and a quite strong luminosity evolution on a time scale $\tau=0.35_{-0.05}^{+0.07}$ $\left(\tau=0.26_{-0.03}^{+0.05}\right)$ for a best-fit $\Lambda$ CDM universe (an empty universe) reference model.

The luminosity function of radio galaxies can be reproduced by a power law with slope in the range $2.4-2.7$ in the luminosity interval $P_{\mathrm{r}}(41 \mathrm{GHz}) \approx 10^{23.3}-10^{27} \mathrm{~W} / \mathrm{Hz}$. As these sources reach the lowest powers, they have the highest number density. Therefore, they are bound to be the dominant non-thermal AGN in the microwave sky at low flux densities. We have measured a value of $\left\langle V / V_{\max }\right\rangle=0.61 \pm 0.07$, consistent with no evolution. However, this is easily explained by a combination of small number statistics (as the sample includes only 15 sources) small redshift range, and a mix of subclasses. A look at the radio morphology of our radio galaxies, from both the literature and the NASA Extragalactic Database (NED), shows a prevalence of FR I sources (9) followed by FR IIs (4), one gigahertz peaked spectrum (GPS) and one compact steep-spectrum object. While FR Is are not understood to be evolving, FR IIs appear to evolve almost as strongly as radio quasars (see Urry \& Padovani 1995 and references therein). We also find a hint of this in our data as well, since $\left\langle V / V_{\max }\right\rangle=0.57 \pm 0.10$ for FR Is and $\left\langle V / V_{\max }\right\rangle=$ $0.67 \pm 0.12$ for non-FR Is.

The present complete sample of microwave-selected nonthermal AGN will allow us to predict the blazar contribution to 
the CMB maps obtainable with coming microwave experiments such as Planck (see e.g., the Planck Blue Book) and Olimpo (Masi et al. 2005). The results obtained from our WMAP sample suggest that FSRQs and BL Lacs will likely dominate the CMB anisotropy spectrum at high microwave fluxes, while other non-thermal AGN of lower flux but higher space density will provide a relevant contribution to the $\mathrm{CMB}$ anisotropies at lower microwave fluxes, if their $\log N-\log S$ relation remains steep, as suggested by previous analyses at radio frequency.

The large number of faint non-blazar AGN will provide a substantial contribution to the diffuse light (effective temperature) of the CMB sky observable with up-coming all-sky surveys. The additional contribution of microwave faint starburst galaxies (which are only marginally present in the high-flux threshold WMAP sample) will probably provide a significant additional contribution to the unresolved $\mathrm{CMB}$ foreground light. We will present a detailed analysis of the contribution of different classes of non-thermal AGN to the cosmic background radiation field in a forthcoming paper (Padovani et al. 2009, in preparation). Detailed considerations about the contribution of the polarized non-thermal AGN to the CMB polarization anisotropy spectrum will also be presented elsewhere (Colafrancesco et al. 2009, in preparation).

All the non-thermal AGNs (blazars, SSRQs and radio galaxies) in our sample are bright, well known sources that have been detected in several energy bands (from radio to X-rays) and are also expected to be detected in the gamma-ray band by the AGILE and Fermi experiments (see below). In this respect, almost 50 WMAP sources already have a counterpart in the third EGRET catalogue of gamma-ray sources (Hartman et al. 1999). In particular, AGILE is expected to detect a similar number of blazars and will enable us to monitor their duty cycle.

Finally, we note that the ratio of the number of FSRQs to $\mathrm{BL}$ Lacs at radio or microwave frequencies is similar to that found at gamma-ray energies. This ratio is $\approx 6$ in our sample (see Table 2), $\approx 8$ in the $5 \mathrm{GHz}$ DXRBS survey Padovani et al. (2007), and $\approx 3-5$ for the EGRET blazars (Sowards-Emmerd et al. 2004).

Since the radio fluxes of EGRET-detected blazars are similar to those of sources detected in the WMAP survey, blazar count ratios can agree only with scenarios where the production of gamma-rays (which is intimately related to radio/microwave photons in Synchrotron - Inverse Compton models) is symmetric with respect to the two blazar types, or slightly favour BL Lacs. Mechanisms such as Synchrotron-Inverse Compton with External components (see, e.g., Sikora \& madjski 2001, for a review), which strongly favour FSRQs cannot therefore be very common otherwise blazars of this type would be far more abundant than BL Lacs at gamma-ray frequencies. The Fermi results to date appear to confirm this. (Abdo et al. 2009) find an FSRQ/BL Lac ratio of 1.3. This relatively low ratio is due to a strong selection effect, which selects radio-faint BL Lacs with synchrotron peak at high energies characterized by a flat gammaray spectrum. By comparing FSRQs and BL Lacs with similar synchrotron peaks (Giommi et al. 2009), one obtains a ratio $\approx 4$, not too different from that derived from the data of WMAP and EGRET. In any case, even Fermi detected no evidence of enhanced gamma-ray emission from FSRQs with respect to that from BL Lacs.
We can conclude that the microwave and gamma-ray energy bands are best suited to study non-thermal AGN since their emission at these frequencies is largely dominated by non-thermal radiation. The combined study of non-thermal AGN in both the microwave and the gamma-ray energy bands will probably offer a unique opportunity to understand many of the physical details of this class of BH-dominated cosmic structures.

Acknowledgements. We are grateful to F. Verrecchia for his technical support in the analysis of the WMAP source confusion. We acknowledge the use of data and software facilities from the ASI Science Data Center (ASDC), managed by the Italian Space Agency (ASI). This work benefits from ASI grant I/024/05/1. Part of this work is based on archival data and bibliographic information obtained from the NASA Extragalactic Database (NED) and from the Astrophysics Data System (ADS).

\section{References}

Abdo, A. A., Ackermann, M., Ajello, M., et al. 2009, ApJ, 700, 597 Avni, Y., \& Bahcall, J. N. 1980, ApJ, 235, 694

Bennett, C. L., Hill, R. S., Hinshaw, G., et al. 2003, ApJS, 148, 97

Blandford, R. D., \& Rees, M. J. 1978, in Pittsburg Conference on BL Lac Objects, ed. A. M. Wolfe (Pittsburgh: University of Pittsburgh press), 328 Crawford, D. F., Jauncey, D. L., \& Murdoch, H. S. 1970, ApJ, 162, 405 Condon, J. J., Cotton, W. D., \& Broderick, J. J. 2002, AJ, 124, 675 Croom, S. M., Smith, R. J., Boyle, B. J., et al. 2004, MNRAS, 349, 1397 Dunlop, J. S., \& Peacock, J. A. 1990, MNRAS, 247, 19 Fanaroff, B. L., \& Riley, J. M. 1974, MNRAS, 167, 31 Gehrels, N. 1986, ApJ, 303, 336

Giommi, P., \& Colafrancesco, S. 2004, A\&A, 414, 7

Giommi, P., \& Colafrancesco, S. 2005, Exp. Astron., 20, 31

Giommi, P., Colafrancesco, S., Cavazzuti, E., et al. 2006, A\&A, 445, 843

Giommi, P., Capalbi, M., Cavazzuti, E., et al. 2007a, A\&A, 468, 571

Giommi, P., Massaro, E., Padovani, P., et al. 2007b, A\&A, 468, 97

Giommi, P., et al. 2009, ApJ, submitted

Grandi, P., \& Palumbo, G. 2004, Sci., 306, 998

Hartman, R. C., Bertsch, D. L., Bloom, S. D., et al. 1999, ApJS, 123, 79

Healey, S. E. Romani, R. W., Taylor, G. B., et al. 2007, ApJS, 171, 61

Healey, S. E., Romani, R. W., Cotter, G., et al. 2008, ApJS, 175, 97

Hinshaw, G., Nolta, M. R., Bennett, C. L., et al. 2007, ApJS, 170, 288

Labiano, A., Barthel, P. D., O’Dea, C. P., et al. 2007, A\&A, 463, 97

Landt, H., Padovani, P., Giommi, P., et al. 2008, ApJ, 676, 87

Marchã, M. J. M., Browne, I. W. A., Impey, C. D., et al. 1996, MNRAS, 281, 425

Massaro, E., Giommi, P., Leto, C., et al. 2009, A\&A, 495, 691

http: //www.asdc.asi.it/bzcat)

Masi, S. et al. 2005, in 17th ESA Symposium on European Rocket and Balloon Programmes and Related Research, Sandefjord, Norway, ed. B. Warmbein, ESA SP-590, Noordwijk: ESA Publications Division, 581

Moorwood, A. F., \& Oliva, E. 1994, ApJ, 429, 602

O'Dea, C. P. 1998, PASP, 110, 493

Padovani, P., \& Urry, M. 1992, ApJ, 387, 449

Padovani, P., Giommi, P., Landt, H., et al. 2007, ApJ, 662, 182

Page, L., Barnes, C., Hinshaw, G., et al. 2003, ApJS, 148, 39

Planck: The Scientific Programme (also known as the Blue Book). European Space Agency. ESA-SCI(2005)-1. Version 2

Schmidt, M. 1968, ApJ, 151, 393

Sikora, M., \& Madejski, G. 2001, in High Energy Gamma-Ray Astronomy, ed.

F. A. Aharonian, \& H. J. Voelk, AIP Conf. proc. 558 (New York: AIP), 275

Sowards-Emmerd, D., Romani, R. W., Michelson, P. F., et al. 2004, ApJ, 609, 564

Spergel, D. N., et al. 2006, ApJ, 148, 175

Spoon, H. W. W., Koornneef, J., Moorwood, A. F. M., et al. 2000, A\&A, 357, 898

Stickel, M., Padovani, P., Urry, C. M., et al. 1991, ApJ, 374, 431

Urry, M., \& Padovani, P. 1995, PASP, 107, 803

Wilkes, B. J., Wright, A. E., Jauncey, D. L., et al. 1983, PASA, 5, 2 